\title{
Serum COX-2 and FOXO3a in patients with rheumatoid arthritis and correlation with disease activity
}

\author{
BANGQIN WANG, XINXIANG HUANG and JINYING LIN \\ Department of Rheumatology, The People's Hospital of Guangxi Zhuang Autonomous Region, \\ Nanning, Guangxi Zhuang Autonomous Region 530021, P.R. China
}

Received April 15, 2019; Accepted October 7, 2019

DOI: $10.3892 /$ etm.2020.8779

\begin{abstract}
Expression levels of serum cyclooxygenase (COX)-2 and forkhead box O3a (FOXO3a) in patients with rheumatoid arthritis (RA) and the correlation with disease activity were investigated. Sixty patients with RA admitted to the People's Hospital of Guangxi Zhuang Autonomous Region (study group; 28 active patients and 32 remissive patients), and further 30 healthy subjects undergoing physical examinations during the same period (control group) were enrolled in this study. RT-qPCR and enzyme-linked immunosorbent assay (ELISA) were used to detect the expression levels of COX-2 and FOXO3a in serum. According to DAS28 score, the patients were divided into active and remissive patients, between whom the expression levels were compared. Receiver operating characteristic (ROC) curves were plotted to analyze the diagnostic values of COX-2 and FOXO3a for disease activity. Pearson's correlation coefficient was used to analyze the correlation of the two markers with erythrocyte sedimentation rate (ESR), C-reactive protein (CRP), and DAS28 score. The expression levels of COX-2 and FOXO3a in active and remissive patients were significantly higher than those in the control group (both $\mathrm{P}<0.05$ ), and those in active patients were significantly higher than those in remissive patients (both $\mathrm{P}<0.05$ ). The areas under the ROC curves (AUCs) of COX-2 and FOXO3a were 0.748 and 0.802 , respectively, suggesting that the two markers have high diagnostic value. The expression levels of COX-2 and FOXO3a were positively correlated with ESR, CRP, and DAS28 score of active and remissive patients (both $\mathrm{P}<0.05$ ). In conclusion, the expression levels of $\mathrm{COX}-2$ and $\mathrm{FOXO} 3 \mathrm{a}$ in patients with RA are upregulated, thus, the two markers may be involved in the development and progression of the disease. The expression levels of COX-2 and FOXO3a are related to the disease activity of RA, and therefore can be used as diagnostic indicators for the disease activity.
\end{abstract}

Correspondence to: Dr Jinying Lin, Department of Rheumatology, The People's Hospital of Guangxi Zhuang Autonomous Region, 6 Taoyuan Road, Nanning, Guangxi Zhuang Autonomous Region 530021, P.R. China

E-mail: ejpp58@163.com; jinyinglin@sina.com

Key words: rheumatoid arthritis, COX-2, FOXO3a, disease activity

\section{Introduction}

Rheumatoid arthritis (RA), an autoimmune disease caused by unclear factors, occurs more commonly among females of $30-50$ years of age (1). Its incidence in China is $0.2-0.4 \%$, and its prevalence ratio of male to female is $1: 3(2,3)$. Clinically, patients with RA experience systemic inflammatory responses that cause an imbalance between pro- and anti-inflammatory cytokines, which results in more immunologic complications if the patients are not treated in time (4). Although remarkable progress has been made in the treatment of RA, the specific mechanism of the disease remains unclear, and the patients suffer great direct and indirect losses (5). Therefore, it is necessary for clinical researchers to explore the pathogenesis of RA and find new therapeutic schemes.

Cyclooxygenase (COX) is an important modulator of prostaglandin synthesis and mainly exists as COX-1 and COX-2 (6). The former is expressed in most tissues and is directly responsible for prostaglandin production (7). The expression of the latter is extremely low in normal tissues and cells, and is massively produced by cells only when induced (8). According to studies, the overexpression of COX-2 inhibits apoptosis (9), and the expression is closely related to the conditions of patients with RA (10). Forkhead box O3a (FOXO3a) belongs to the forkhead family of transcription factors, and its differential expression is closely associated with the proliferation of helper T cells (11). There are numerous studies on FOXO3a in tumors, however, there are few studies on FOXO3a in RA. For example, FOXO3a controls the development and progression of tumors by regulating related proteins (PUMA and Noxa) in lymphocytes and neuroblastoma. The detected FOXO3a expression can be used as a prognostic marker for patients with breast cancer $(12,13)$. The dysfunction of FOXO3a aggravates arthritis (14). However, the correlation of FOXO3a with patients' disease activity remains unclear.

Therefore, the expression levels of FOXO3a and COX-2 and their correlation with the disease activity of patients with RA were explored in this study to provide new targets for the treatment of the disease.

\section{Patients and methods}

Clinical data. Sixty patients with RA admitted to the People's Hospital of Guangxi Zhuang Autonomous Region (Nanning, 
China) from May 2016 to May 2017 (study group) were enrolled in this study. The patients consisted of 16 males and 44 females, with an average age of $60.4 \pm 10.7$ years. Further 30 healthy subjects undergoing physical examinations in the hospital during the same period (control group) were also enrolled. The healthy subjects had normal biochemical, blood routine, immunological and microbial indicators, without congenital immunodeficiency. The study was approved by the Medical Ethics Committee of the People's Hospital of Guangxi Zhuang Autonomous Region. Inclusion criteria: Patients who met the diagnostic criteria for RA of the European League Against Rheumatism of the American College of Rheumatology in 2009 (15); patients with complete clinical data; patients who were evaluated by DAS28 score; patients who they or their families were informed and signed an informed consent form; active patients who were initially diagnosed; stable patients who had been confirmed and who were in the remissive stage after treatment. Exclusion criteria: Patients $<18$ years of age; patients with tumors; patients with other congenital immunodeficiency diseases; patients with congenital defects of heart, lung, or brain; patients who were unsound on their feet; active patients who had taken hormone and immunosuppressive drugs before treatment.

Sources of kits. COX-2 enzyme-linked immunosorbent assay (ELISA) kit (mlbio - Shanghai Enzyme-linked Biotechnology Co., Ltd.; cat. no. m1062904), C-reactive protein (CRP) kit (Shanghai Tellgen Life Science Co., Ltd.; BH031), TRIzol total RNA extraction reagent (Invitrogen; Thermo Fisher Scientific, Inc.; 15596018 and N8080234, respectively), PrimeScript $^{\mathrm{TM}}$ RT reagent kit (Takara Bio, Inc.; RR037A). Primer sequences were designed and synthesized by Sangon Biotech Co., Ltd. PCR instrument (Applied Biosystems; Thermo Fisher Scientific, Inc.; ABI 7500).

Sample collection. Fasting peripheral venous blood $(10 \mathrm{ml})$ was respectively extracted from the patients of the study and the control group, and was distributed into 4 tubes. The blood in the 3 tubes was allowed to stand for $30 \mathrm{~min}$, and then, it was centrifuged at $1,509 \times \mathrm{g}$ at $25^{\circ} \mathrm{C}$ for $10 \mathrm{~min}$ to collect the supernatant for subsequent experiments. The blood in the other tube was detected for erythrocyte sedimentation rate (ESR).

Detection of COX-2. The collected serum was detected using ELISA. Specific steps were as follows: $50 \mu \mathrm{l}$ of serum and sample diluent (1:1), respectively, were added to a 96-well plate. Blank and standard wells were set up, and $50 \mu 1$ of biotin-labeled antibodies were added to each well. The ELISA plate was sealed, incubated at $37^{\circ} \mathrm{C}$ for $1 \mathrm{~h}$, and then washed. Next, streptavidin-HRP monoclonal antibody $(80 \mu 1)$ was added to each well and after incubation at $37^{\circ} \mathrm{C}$ for $30 \mathrm{~min}$ the wells were washed. Substrates A and B (50 $\mu$ l each) were respectively added to each well and incubation followed at $37^{\circ} \mathrm{C}$ for $10 \mathrm{~min}$ in the dark. Finally, stop solution $(50 \mu \mathrm{l})$ was added to each well. Optical density (OD) values at $450 \mathrm{~nm}$ were detected using a microplate reader within $15 \mathrm{~min}$. The experiment was repeated 3 times to obtain the average value.

Detection of FOXO3a. TRIzol reagent was used to extract total RNA from the collected serum. UV spectrophotometer and agarose gel electrophoresis were used to detect its purity, concentration and integrity. Total RNA was reverse transcribed using the PrimeScript ${ }^{\mathrm{TM}}$ RT reagent kit and cDNA was collected for subsequent experiments, with the operation steps carried out according to the manufacturer's instructions. The specific steps were as follows: $4 \mu \mathrm{l}$ of $5 \mathrm{X}$ RT buffer, $2 \mu \mathrm{l}$ of $10 \mathrm{mM}$ dNTPs, $0.4 \mu \mathrm{l}$ of RNasin $(40 \mathrm{U} / \mu \mathrm{l}), 1 \mu \mathrm{l}$ of M-MLVRTase $(200 \mathrm{U} / \mu \mathrm{l})$, and RNase free $\mathrm{H}_{2} \mathrm{O}$ was added to a final volume of $25 \mu \mathrm{l}$. The PCR amplification system was as follows: $2 \mu \mathrm{l}$ of cDNA, each $0.8 \mu \mathrm{l}$ of upstream and downstream primers, $10 \mu \mathrm{l}$ of TB Green Premix Ex Taq II (Tli RNaseH Plus) (2X), $0.4 \mu 1$ of ROX Reference Dye or Dye II (50X), and $\mathrm{ddH}_{2} \mathrm{O}$ was finally added to a final volume of $20 \mu \mathrm{l}$. The TaqMan $^{\mathrm{TM}}$ reverse transcription reagent kit was used for TB Green Premix Ex Taq II (Tli RNaseH Plus) and ROX Reference Dye or Dye II. The thermocycling conditions were as follows: Pre-denaturation at $95^{\circ} \mathrm{C}$ for $30 \mathrm{sec}$, denaturation at $95^{\circ} \mathrm{C}$ for $5 \mathrm{sec}$, annealing and extension at $60^{\circ} \mathrm{C}$ for $34 \mathrm{sec}$, for 40 cycles. Each sample was provided with 3 identical wells, and the experiment was carried out 3 times. GAPDH was used as an internal reference and $2^{-\Delta \mathrm{Cq}}$ was used to analyze the data (16). The upstream and downstream primers of FOXO3a were 5'-AAGCCAGCTACCTTCTCTTCCA-3' and 5'-CTGGCT AAGTGAGTCCGAAGTGA-3', respectively, and of GAPDH were 5'-CACCCACTCCTCCACCTTTG-3' and 5'-CCACCA CCCTGTTGCTGTAG-3', respectively.

Detection of ESR and CRP. ESR was detected by an automatic ESR analyzer (Vital Diagnostics), and CRP was detected by a Hitachi 7600 fully automatic biochemical analyzer (Hitachi, Ltd.) using immune nephelometry, in strict accordance with the manufacturer's instructions.

Observational indices. Main observational indices: The expression levels of serum COX-2 and FOXO3a in the control and study groups were observed. According to the DAS28 score, the patients were divided into active and remissive patients ( $\geq 2.6$ points for active stage and $<2.6$ points for remissive stage), between whom the expression levels were compared.

Secondary observational indices: Receiver operating characteristic (ROC) curves were plotted to analyze the diagnostic values of COX-2 and FOXO3a for disease activity. Pearson's correlation coefficient was used to analyze the correlation of the two markers with ESR, CRP, and DAS28 score.

Statistical analysis. SPSS 20.0 (Cabit Information Technology Co., Ltd.) was used to statistically analyze the data. GraphPad Prism 7 (Softhead, Inc.) was used to plot figures. Count data were expressed as percentage (\%), analyzed by Chi-square test, and represented by $\chi^{2}$. Kolmogorov-Smirnov test was used to analyze the data distribution. Measurement data were expressed by mean \pm standard deviation (means \pm SD). The data conforming to normal distribution were analyzed by independent samples t-test, and represented by t. Comparison between multiple groups was made by one-way analysis of variance and represented by F. Pairwise comparison between groups was analyzed by univariate LSD-t test. Pearson's correlation coefficient was used for analyzing the relationship between indices. ROC curves were plotted to analyze the diagnostic values of COX-2 and FOXO3a for disease activity. $\mathrm{P}<0.05$ was considered to indicate a statistically significant difference. 
Table I. Baseline data.

\begin{tabular}{|c|c|c|c|c|}
\hline Factors & Study group $(n=60)$ & Control group $(n=30)$ & $\mathrm{t} / \chi^{2}$ value & P-value \\
\hline Sex & & & 0.433 & 0.511 \\
\hline Male & $16(26.67)$ & $10(33.33)$ & & \\
\hline Female & $44(73.33)$ & $20(66.67)$ & & \\
\hline Age (years) & $60.4 \pm 10.7$ & $58.7 \pm 8.9$ & 0.750 & 0.455 \\
\hline $\operatorname{BMI}\left(\mathrm{kg} / \mathrm{m}^{2}\right)$ & $22.04 \pm 1.77$ & $22.59 \pm 1.80$ & 1.382 & 0.171 \\
\hline \multicolumn{5}{|l|}{ Past medical history } \\
\hline Hypertension & $19(31.67)$ & $8(26.67)$ & 0.238 & 0.626 \\
\hline Diabetes & $22(36.67)$ & $10(33.33)$ & 0.097 & 0.756 \\
\hline Hyperlipemia & $12(20.00)$ & $5(16.67)$ & 0.145 & 0.703 \\
\hline History of smoking & & & 0.407 & 0.524 \\
\hline Yes & $18(30.00)$ & $11(36.67)$ & & \\
\hline No & $42(70.00)$ & $19(63.33)$ & & \\
\hline History of alcoholism & & & 0.078 & 0.781 \\
\hline Yes & $5(8.33)$ & $2(6.67)$ & & \\
\hline No & $55(91.67)$ & $28(93.33)$ & & \\
\hline Place of residence & & & 0.215 & 0.643 \\
\hline City & $37(61.67)$ & $20(66.67)$ & & \\
\hline Countryside & $23(38.33)$ & $10(33.33)$ & & \\
\hline Course of disease (years) & $8.54 \pm 2.11$ & & & \\
\hline $\mathrm{CRP}(\mathrm{mg} / \mathrm{l})$ & $53.84 \pm 22.06$ & $5.81 \pm 2.87$ & 11.842 & $<0.001$ \\
\hline $\operatorname{ESR}(\mathrm{mm} / \mathrm{h})$ & $33.15 \pm 18.22$ & $9.88 \pm 4.45$ & 6.876 & $<0.001$ \\
\hline DAS28 score & $3.12 \pm 1.45$ & & & \\
\hline
\end{tabular}

BMI, body mass index; CRP, C-reactive protein; ESR, erythrocyte sedimentation rate.
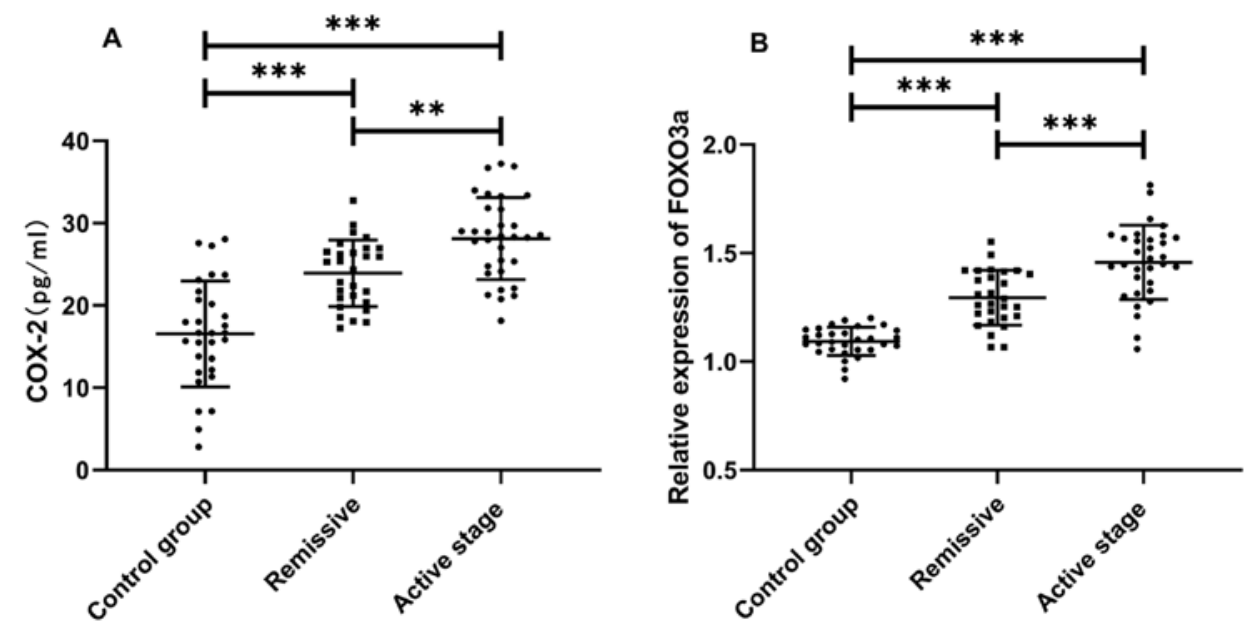

Figure 1. Expression levels of COX-2 and FOXO3a in the study group. (A) COX-2 was highly expressed in patients with RA. The expression of COX-2 in active patients was higher than that in remissive patients. (B) FOXO3a was highly expressed in patients with RA. The expression of FOXO3a in active patients was higher than that in remissive patients. ${ }^{* *} \mathrm{P}<0.01,{ }^{* * *} \mathrm{P}<0.001$. COX, cyclooxygenase; FOXO3a, forkhead box O3a; RA, rheumatoid arthritis.

\section{Results}

Clinical data. There were statistically significant differences between the study and the control group in terms of CRP and ESR $(\mathrm{P}<0.05)$, but not in terms of sex, age, body mass index (BMI), past medical history, history of smoking, history of alcoholism, and place of residence ( $\mathrm{P}>0.05)$ (Table I).

Expression levels of $\mathrm{COX}-2$ and FOXO3a in the study and the control group. According to the detection results, the expression 
Table II. ROC parameters.

\begin{tabular}{lcccccc}
\hline Indicators & AUC & $95 \% \mathrm{CI}$ & Specificity $(\%)$ & Sensitivity $(\%)$ & Youden index $(\%)$ & Cut-off \\
\hline COX-2 & 0.748 & $0.624-0.871$ & 62.50 & 85.71 & 48.21 & $<27.671 \mathrm{pg} / \mathrm{ml}$ \\
FOXO3a & 0.802 & $0.689-0.916$ & 68.75 & 92.85 & 61.61 & $<1.424$ \\
CRP & 0.708 & $0.613-0.803$ & 80.00 & 65.00 & 45.00 & $<7.25 \mathrm{mg} / 1$ \\
ESR & 0.702 & $0.609-0.794$ & 86.67 & 46.67 & 33.33 & $<14.85 \mathrm{~mm} / \mathrm{h}$
\end{tabular}

ROC, receiver operating characteristic; AUC, area under the curve; 95\% CI, 95\% confidence interval; Cut-off, best cut-off value; COX, cyclooxygenase; FOXO3a, forkhead box O3a; CRP, C-reactive protein; ESR, erythrocyte sedimentation rate.

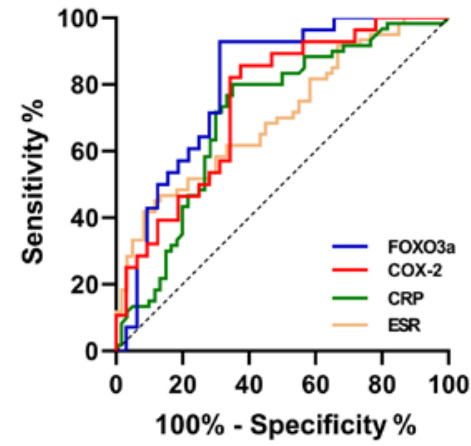

Figure 2. ROC curves. Blue line represents FOXO3a and red line represents COX-2. Green line represents CRP and the light brown line represents ESR. ROC, receiver operating characteristic; COX, cyclooxygenase; FOXO3a, forkhead box O3a; CRP, C-reactive protein; ESR, erythrocyte sedimentation rate

levels of COX-2 and FOXO3a in the study group were significantly higher than those in the control group $(\mathrm{P}<0.05)$. The comparison of COX-2 and FOXO3a expression levels between the active and remissive patients showed that the expression levels in active patients were significantly higher than those in remissive patients $(\mathrm{P}<0.05)$ (Fig. 1).

Diagnostic values of COX-2 and FOXO3a for disease activity. According to the ROC curves, the area under the curve (AUC) of COX-2 was 0.748 . When the specificity was $62.50 \%$ and the sensitivity was $85.71 \%$, the best cut-off value of COX-2 was $27.671 \mathrm{pg} / \mathrm{ml}$. The AUC of FOXO3a was 0.802 . When the specificity was $68.75 \%$ and the sensitivity was $92.85 \%$, the best cut-off value of FOXO3a was 1.424. The AUC of CRP was 0.708 . When the specificity was $80.00 \%$ and the sensitivity was $65.00 \%$, the best cut-off value of CRP was $7.25 \mathrm{mg} / \mathrm{l}$. The AUC of ESR was 0.702 . When the specificity was $86.67 \%$ and the sensitivity was $46.67 \%$, the best cut-off value of ESR was $14.85 \mathrm{~mm} / \mathrm{h}$ (Table II and Fig. 2).

Correlation of COX-2 and FOXO3a with ESR, CRP, and DAS28 score. According to Pearson's correlation coefficient, the expression levels of COX-2 and FOXO3a were positively correlated with ESR, CRP, and DAS28 score of active and remissive patients (both $\mathrm{P}<0.05$ ) (Tables III and IV, and Fig. 3).

\section{Discussion}

$\mathrm{RA}$ is the most common joint disease in clinical practice. Systemic inflammatory responses stimulate the proliferation
Table III. Correlation of COX-2 with ESR, CRP, and DAS28 score.

\begin{tabular}{lrrrrr}
\hline & \multicolumn{2}{c}{$\begin{array}{c}\text { Active patients } \\
(\mathrm{n}=28)\end{array}$} & & \multicolumn{2}{c}{$\begin{array}{c}\text { Remissive patients } \\
(\mathrm{n}=32)\end{array}$} \\
\cline { 2 - 3 } \cline { 5 - 6 } Factors & r value & P-value & & r value & P-value \\
\hline ESR & 0.618 & $<0.001$ & & 0.773 & $<0.001$ \\
CRP & 0.551 & 0.001 & & 0.551 & 0.002 \\
DAS28 & 0.669 & $<0.001$ & & 0.658 & $<0.001$ \\
\hline
\end{tabular}

COX, cyclooxygenase; ESR, erythrocyte sedimentation rate; CRP, C-reactive protein.

Table IV. Correlation of FOXO3a with ESR, CRP, and DAS28 score.

\begin{tabular}{lrrrrr}
\hline & \multicolumn{2}{c}{$\begin{array}{c}\text { Active patients } \\
(\mathrm{n}=28)\end{array}$} & & \multicolumn{2}{c}{$\begin{array}{c}\text { Remissive patients } \\
(\mathrm{n}=32)\end{array}$} \\
\cline { 2 - 3 } Factors & r value & P-value & & r value & P-value \\
\hline ESR & 0.605 & $<0.001$ & & 0.525 & 0.004 \\
CRP & 0.719 & $<0.001$ & & 0.753 & $<0.001$ \\
DAS28 & 0.460 & 0.008 & & 0.626 & $<0.001$ \\
\hline
\end{tabular}

FOXO3a, forkhead box O3a; ESR, erythrocyte sedimentation rate; CRP, C-reactive protein.

of synovial cells, cause the formation of invasive pannus, and damage the cartilage and bone tissue, thereby leading to the disease (17). Statistics have shown that the disability rate of RA in China is high, and the disease seriously affects the patients' daily life and work (18). The disease is currently treated with anti-inflammatory agents and analgesics, both of which only relieve patients' conditions, but cannot cure the disease (19). Therefore, it is essential to find new therapeutic targets to improve the conditions.

In this study, the expression of COX-2 in the study group was significantly higher than that in the control group. A large number of studies have shown that COX-2 is highly expressed in patients with RA $(20,21)$, which is consistent with our 

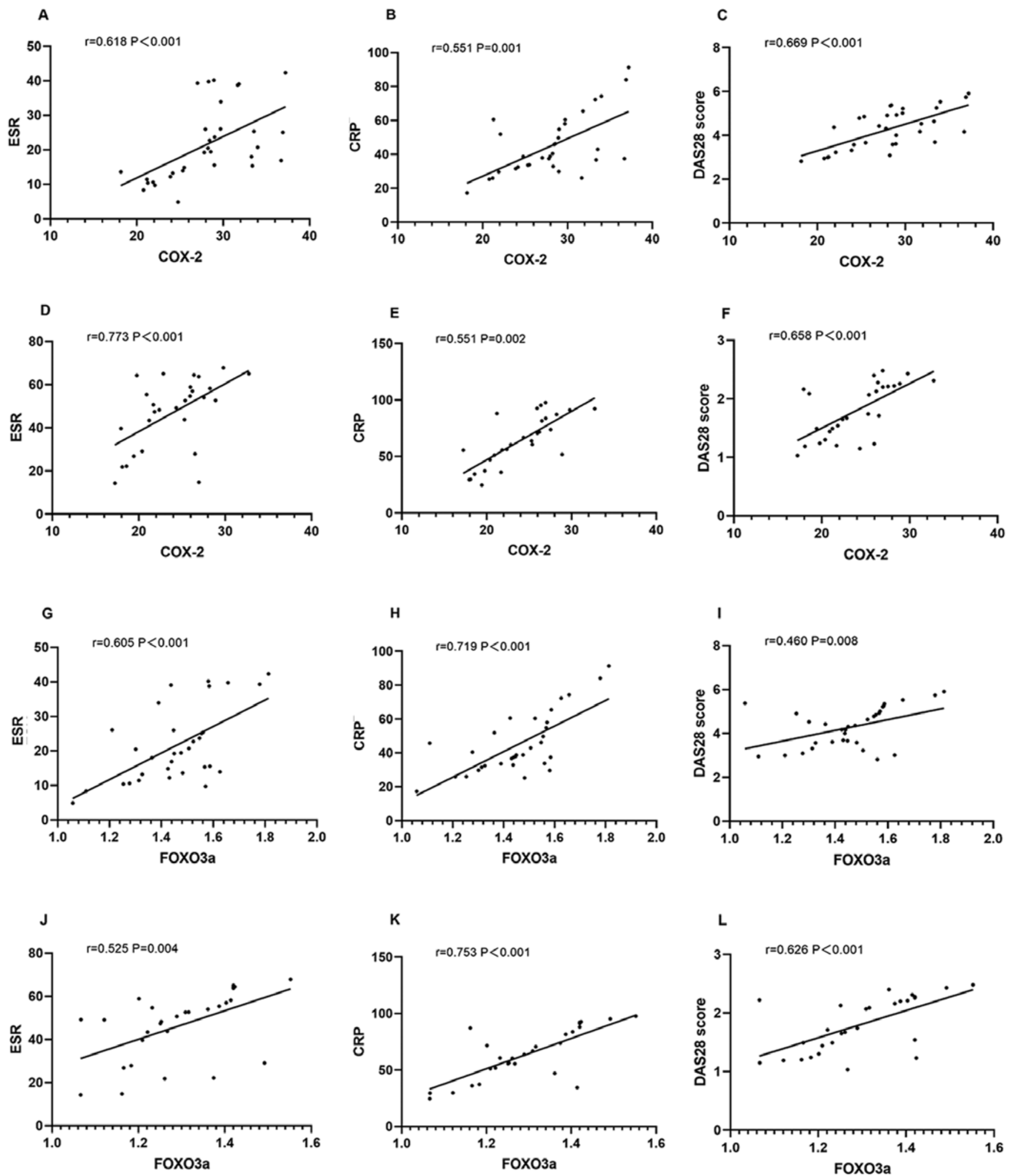

Figure 3. Correlation of COX-2 and FOXO3a with ESR, CRP, and DAS28 score. The correlation of COX-2 with (A) ESR, (B) CRP, and (C) DAS28 score of active patients is presented. The correlation of COX-2 with (D) ESR, (E) CRP, and (F) DAS28 score of remissive patients is presented. The correlation of FOXO3a with (G) ESR, (H) CRP, and (I) DAS28 score of active patients is presented. The correlation of FOXO3a with (J) ESR, (K) CRP, and (L) DAS28 score of remissive patients is presented. COX, cyclooxygenase; FOXO3a, forkhead box O3a; ESR, erythrocyte sedimentation rate; CRP, C-reactive protein.

findings. Therefore, COX-2 inhibitors have been widely used in clinical practice, especially in the treatment of RA $(22,23)$. As a subfamily member of forkhead transcription factors, FOXO3a mediates apoptosis, cell proliferation, cell cycle progression, DNA damage, and tumorigenesis (24). However, there are few studies on FOXO3a in RA. In this study, the expression of FOXO3a in the study group was found to be significantly higher than that in the control group, indicating 
that FOXO3a is differentially expressed in patients with RA and healthy subjects. In a study by Turrel-Davin et al (25), the expression of serum FOXO3a in patients with RA, as detected by microarray chips, was significantly higher than that in healthy subjects, which is consistent with our findings.

According to different disease activities, the patients were divided into active and remissive patients. The expression levels of COX-2 and FOXO3a in remissive patients were significantly lower than those in active patients. According to Turrel-Davin et al (25), the overexpression of FOXO3a promotes the proliferation and survival of neutrophils and synovial $\mathrm{T}$ cells in the peripheral blood of patients with RA. Therefore, the conditions of active patients were more severe than those of remissive patients, possibly because the promotion of the overexpression aggravates the patients' conditions. However, it is still unclear whether COX-2 and FOXO3a can be used as markers to distinguish active patients from remissive patients. According to the ROC curves, the AUCs of COX-2 and FOXO3a were 0.748 and 0.802 , respectively, slightly higher than those of ESR and CRP, indicating that COX-2 and FOXO3a have good diagnostic values for active and remissive patients.

Finally, the correlation of COX-2 and FOXO3a with ESR, CRP, and DAS28 score of active and remissive patients were analyzed. DAS28 score, the accuracy of which has been confirmed in a number of tests, is an internationally recognized standard for evaluating the conditions of patients with RA through laboratory parameters and swollen joint count (26). CRP is not the best specific indicator, however, is an important laboratory parameter for RA activity, because its increase directly reflects inflammation (27). ESR is an important index for the clinical observation of RA. Rouleaux formation is easy to occur in erythrocytes due to the massive production of inflammatory cytokines in patients, thereby accelerating ESR (28). According to Pearson's correlation coefficient, COX-2 and FOXO3a were shown to be positively correlated the ESR, CRP, and DAS28 score of active and remissive patients, which suggests that $\mathrm{COX}-2$ and $\mathrm{FOXO} 3 \mathrm{a}$ may relieve the patients' conditions.

The present study proves that COX-2 and FOXO3a are highly expressed in patients with RA, and that the two markers can be used as potential indicators for distinguishing different disease activities. However, there are still limitations. The expression levels of COX-2 and FOXO3a in the synovial tissue of the patients were not detected. The relationship between FOXO3a and COX-2 was not fully explored in this clinical study. The mechanism of action of FOXO3a in RA is also unclear. The expression levels of the two markers in the patients' serum were detected, however, the patients were not followed up. Therefore, further study is still required.

In conclusion, the expression levels of COX-2 and FOXO3a in patients with RA are upregulated, so the two markers may be involved in the development and progression of the disease. The expression levels of COX-2 and FOXO3a are related to the disease activity of RA, and thus, they can be used as diagnostic indicators.

\section{Acknowledgements}

Not applicable.

\section{Funding}

No funding was received.

\section{Availability of data and materials}

The datasets used and/or analyzed during the present study are available from the corresponding author on reasonable request.

\section{Authors' contributions}

BW wrote the manuscript. BW and $\mathrm{XH}$ performed PCR and ELISA. JL analyzed and interpreted the patient data, and assisted with statistical analysis. All authors read and approved the final manuscript.

\section{Ethics approval and consent to participate}

The study was approved by the Medical Ethics Committee of the People's Hospital of Guangxi Zhuang Autonomous Region (Nanning, China). Patients who participated in this research signed an informed consent and had complete clinical data.

\section{Patient consent for publication}

Not applicable.

\section{Competing interests}

The authors declare that they have no competing interests.

\section{References}

1. Singh JA, Saag KG, Bridges SL Jr, Akl EA, Bannuru RR, Sullivan MC, Vaysbrot E, McNaughton C, Osani M, Shmerling RH, et al: 2015 American College of Rheumatology Guideline for the Treatment of Rheumatoid Arthritis. Arthritis Rheumatol 68: 1-26, 2016.

2. Jin S, Li M, Fang Y, Li Q, Liu J, Duan X, Liu Y, Wu R, Shi X, Wang Y, et al; CREDIT Co-authors: Chinese Registry of rheumatoid arthritis (CREDIT): II. prevalence and risk factors of major comorbidities in Chinese patients with rheumatoid arthritis. Arthritis Res Ther 19: 251, 2017.

3. de Achaval S and Suarez-Almazor ME: Treatment adherence to disease-modifying antirheumatic drugs in patients with rheumatoid arthritis and systemic lupus erythematosus. Int J Clin Rheumatol 5: 313-326, 2010.

4. Malmström V, Catrina AI and Klareskog L: The immunopathogenesis of seropositive rheumatoid arthritis: From triggering to targeting. Nat Rev Immunol 17: 60-75, 2017.

5. Catrina AI, Joshua V, Klareskog L and Malmström V: Mechanisms involved in triggering rheumatoid arthritis. Immunol Rev 269: $162-174,2016$.

6. Zelenay S, van der Veen AG, Böttcher JP, Snelgrove KJ, Rogers N, Acton SE, Chakravarty P, Girotti MR, Marais R, Quezada SA, et al: Cyclooxygenase-dependent tumor growth through evasion of immunity. Cell 162: 1257-1270, 2015.

7. Depboylu C, Weihe E and Eiden LE: COX1 and COX2 expression in non-neuronal cellular compartments of the rhesus macaque brain during lentiviral infection. Neurobiol Dis 42: 108-115, 2011.

8. Yang CM, Chen YW, Chi PL, Lin CC and Hsiao LD: Resveratrol inhibits BK-induced COX-2 transcription by suppressing acetylation of AP-1 and NF- $\kappa$ B in human rheumatoid arthritis synovial fibroblasts. Biochem Pharmacol 132: 77-91, 2017.

9. Walther U, Emmrich K, Ramer R, Mittag N and Hinz B: Lovastatin lactone elicits human lung cancer cell apoptosis via a COX-2/PPAR $\gamma$-dependent pathway. Oncotarget 7: 10345-10362, 2016. 
10. Lowin T, Apitz M, Anders S and Straub RH: Anti-inflammatory effects of N-acylethanolamines in rheumatoid arthritis synovial cells are mediated by TRPV1 and TRPA1 in a COX-2 dependent manner. Arthritis Res Ther 17: 321, 2015.

11. Tang YL, Huang LB, Lin WH, Wang LN, Tian Y, Shi D, Wang J, Qin G, Li A, Liang YN, et al: Butein inhibits cell proliferation and induces cell cycle arrest in acute lymphoblastic leukemia via FOXO3a/p27kipl pathway. Oncotarget 7: 18651-18664, 2016.

12. Obexer P, Geiger K, Ambros PF, Meister B and Ausserlechner MJ: FKHRL1-mediated expression of Noxa and Bim induces apoptosis via the mitochondria in neuroblastoma cells. Cell Death Differ 14: 534-547, 2007.

13. Jiang Y,Zou L, Lu WQ, Zhang Y and Shen AG: Foxo3a expression is a prognostic marker in breast cancer. PLoS One 8: e70746, 2013.

14. Viatte S, Lee JC, Fu B, Espéli M, Lunt M, De Wolf JN, Wheeler L, Reynolds JA, Castelino M, Symmons DP, et al: Association between genetic variation in FOXO3 and reductions in inflammation and disease activity in inflammatory polyarthritis. Arthritis Rheumatol 68: 2629-2636, 2016.

15. Aletaha D, Neogi T, Silman AJ, Funovits J, Felson DT, Bingham CO III, Birnbaum NS, Burmester GR, Bykerk VP, Cohen MD, et al: 2010 Rheumatoid arthritis classification criteria: An American College of Rheumatology/European League Against Rheumatism collaborative initiative. Arthritis Rheum 62 2569-2581, 2010

16. Livak KJ and Schmittgen TD: Analysis of relative gene expression data using real-time quantitative PCR and the 2(-Delta Delta C(T)) Method. Methods 25: 402-408, 2001.

17. Narayan N, Owen DR, Mandhair H, Smyth E, Carlucci F, Saleem A, Gunn RN, Rabiner EA, Wells L, Dakin SG, et al: Translocator protein as an imaging marker of macrophage and stromal activation in rheumatoid arthritis pannus. J Nucl Med 59: $1125-1132,2018$.

18. Gong $\mathrm{G}$ and Mao J: Health-related quality of life among Chinese patients with rheumatoid arthritis: The predictive roles of fatigue, functional disability, self-efficacy, and social support. Nurs Res 65: 55-67, 2016.

19. Smith SR, Deshpande BR, Collins JE, Katz JN and Losina E: Comparative pain reduction of oral non-steroidal anti-inflammatory drugs and opioids for knee osteoarthritis: Systematic analytic review. Osteoarthritis Cartilage 24: 962-972, 2016.

20. Fan HW, Liu GY, Zhao CF, Li XF and Yang XY: Differential expression of COX-2 in osteoarthritis and rheumatoid arthritis. Genet Mol Res 14: 12872-12879, 2015.
21. Peng A, Lu X, Huang J, He M, Xu J, Huang H and Chen Q: Rheumatoid arthritis synovial fibroblasts promote TREM-1 expression in monocytes via COX-2/PGE 2 pathway. Arthritis Res Ther 21: 169, 2019.

22. Roubille C, Richer V, Starnino T, McCourt C, McFarlane A Fleming P, Siu S, Kraft J, Lynde C, Pope J, et al: The effects of tumour necrosis factor inhibitors, methotrexate, non-steroidal anti-inflammatory drugs and corticosteroids on cardiovascular events in rheumatoid arthritis, psoriasis and psoriatic arthritis: A systematic review and meta-analysis. Ann Rheum Dis 74: 480-489, 2015.

23. Nissen SE, Yeomans ND, Solomon DH, Lüscher TF, Libby P, Husni ME, Graham DY, Borer JS, Wisniewski LM, Wolski KE, et al; PRECISION Trial Investigators: Cardiovascular safety of celecoxib, naproxen, or ibuprofen for arthritis. N Engl J Med 375: 2519-2529, 2016.

24. Codogno P and Morel E: FOXO3a provides a quickstep from autophagy inhibition to apoptosis in cancer therapy. Dev Cell 44: 537-539, 2018.

25. Turrel-Davin F, Tournadre A, Pachot A, Arnaud B, Cazalis MA, Mougin B and Miossec P: FoxO3a involved in neutrophil and T cell survival is overexpressed in rheumatoid blood and msynovial tissue. Ann Rheum Dis 69: 755-760, 2010.

26. Yoshida K, Matsui K, Nakano H, Oshikawa H, Utsunomiya M, Kobayashi T, Kimura M, Deshpande GA and Kishimoto M: Response to "Pain persists in DAS28 rheumatoid arthritis remission but not in ACR/EULAR remission: A longitudinal observational study'. Arthritis Res Ther 13: 405, 2011

27. Sheehy C, Evans V, Hasthorpe H and Mukhtyar C: Revising DAS28 scores for remission in rheumatoid arthritis. Clin Rheumatol 33: 269-272, 2014.

28. Shimada K, Komiya A, Yokogawa N, Nishino J, Sugii S and Tohma S: Impact of the size and number of swollen joints on serum C-reactive protein level and erythrocyte sedimentation rate in rheumatoid arthritis: A cross-sectional study in Japan. Clin Rheumatol 36: 427-431, 2017.

This work is licensed under a Creative Commons Attribution-NonCommercial-NoDerivatives 4.0 International (CC BY-NC-ND 4.0) License. 